\title{
Differential adsorption of complex organic molecules isomers at interstellar ice surfaces
}

\author{
M. Lattelais ${ }^{1}$, M. Bertin ${ }^{2}$, H. Mokrane ${ }^{3}$, C. Romanzin ${ }^{2}$, X. Michaut ${ }^{2}$, P. Jeseck ${ }^{2}$, J.-H. Fillion ${ }^{2}$, H. Chaabouni ${ }^{3}$, \\ E. Congiu ${ }^{3}$, F. Dulieu ${ }^{3}$, S. Baouche ${ }^{3}$, J.-L. Lemaire ${ }^{3}$, F. Pauzat, ${ }^{1}$, J. Pilmé ${ }^{1}$, C. Minot $^{1}$, and Y. Ellinger ${ }^{1}$ \\ 1 UPMC Univ. Paris 06, UMR - CNRS 7616, Laboratoire de Chimie Théorique (LCT), 75005 Paris, France \\ e-mail: pauzat@lct.jussieu.fr \\ 2 UPMC Univ. Paris 06, UMR - CNRS 7092, Laboratoire de Physique Moléculaire pour l'Atmosphère et l'Astrophysique (LPMAA), \\ 75005 Paris, France \\ e-mail: jean-hugues . fillion@upmc.fr \\ 3 Observatoire de Paris et Université de Cergy Pontoise, UMR-CNRS 8112, Laboratoire d'Étude du Rayonnement et de la Matière \\ en Astrophysique/Laboratoire Atomes et Molécules en Astrophysique (LERMA/LAMAp), 95000 Cergy Pontoise, France \\ e-mail: jean-louis.lemaire@obspm.fr
}

Received 23 November 2010 / Accepted 7 May 2011

\section{ABSTRACT}

\begin{abstract}
Context. Over 20 of the $\sim 150$ different species detected in the interstellar and circumstellar media have also been identified in icy environments. For most of the species observed so far in the interstellar medium (ISM), the most abundant isomer of a given generic chemical formula is the most stable one (minimum energy principle - MEP) with few exceptions such as, for example, $\mathrm{CH}_{3} \mathrm{COOH} / \mathrm{HCOOCH}_{3}$ and $\mathrm{CH}_{3} \mathrm{CH}_{2} \mathrm{OH} / \mathrm{CH}_{3} \mathrm{OCH}_{3}$, whose formation is thought to occur on the icy mantles of interstellar grains. Aims. We investigate whether differences found in the compositions of molecular ices and the surrounding gas phase could originate from differences between the adsorption of one isomer from that of another at the ice surface.

Methods. We performed a coherent and concerted theoretical/experimental study of the adsorption energies of the four molecules mentioned above, i.e. acetic acid (AA)/methyl formate (MF) and ethanol (EtOH)/dimethyl ether (DME) on the surface of water ice at low temperature. The question was first addressed theoretically at LCT using solid state periodic density functional theory (DFT) to represent the organized solid support. The experimental determination of the ice/molecule interaction energies was then carried out independently by two teams at LPMAA and LERMA/LAMAp using temperature programmed desorption (TPD) under an ultra-high vacuum (UHV) between 70 and $160 \mathrm{~K}$.

Results. For each pair of isomers, theory and experiments both agree that the most stable isomer (AA or EtOH) interacts more efficiently with the water ice than the higher energy isomer (MF or DME). This differential adsorption can be clearly seen in the different desorption temperatures of the isomers. It is not related to their intrinsic stability but instead to both AA and EtOH producing more and stronger hydrogen bonds with the ice surface.

Conclusions. We show that hydrogen bonding may play an important role in the release of organic species from grains and propose that, depending on the environment, differential adsorption should not be rejected as a possible way of interpreting MEP exceptions.
\end{abstract}

Key words. astrochemistry - ISM: abundances - ISM: molecules

\section{Introduction}

It is well known that gas phase chemistry alone cannot account for the diversity of complex organic molecules observed in the interstellar medium (ISM) and that solid-gas reactions, and even chemical processes involving adsorbed partners, have to be considered. These small particles may protect the molecules from the radiation field and, at the same time, play the role of a thermodynamic bath acting as a third body to dissipate the energy excess of exothermic reactions. They may also provide the catalytic conditions to overcome small barriers to chemical reaction paths and/or directly contribute to the reaction budget.

Over the past 35 years, a number of grain models have been proposed. They include refractory minerals, such as silicates and metal oxides (possibly mixed with graphitic carbon Mathis et al. 1977) and ices and/or carbonaceous coatings on mineral particles (Greenberg 1976). Following these basic ideas, various components were later identified, mainly from mid-infrared spectroscopy, which is one of the most helpful techniques for probing solid materials (Tielens \& Allamandola 1987).
Examples include carbonates (Kemper et al. 2002; Chiavassa et al. 2005), silicates (Molster et al. 2002) and complex interstellar ices (Sandford 1996; Allamandola et al. 1999; Schutte 1999; Gibb et al. 2004). From a chemical point of view, the case of ices (Watanabe \& Kouchi 2008) is particularly interesting for the synthesis of oxygen-containing molecules such as for example $\mathrm{H}_{2} \mathrm{O}, \mathrm{CO}_{2}, \mathrm{H}_{2} \mathrm{CO}, \mathrm{CH}_{3} \mathrm{OH}$, or glycine isomers (Watanabe et al. 2003, 2004; Holtom et al. 2005; Bossa et al. 2008, 2009; Dulieu et al. 2010).

However, the precise composition and morphology of interstellar grains, as well as the identification of the constituents of their icy mantles, remains unclear. As a consequence, any modelling of grain surfaces is also a matter of discussion and the theoretical results have to be confronted with laboratory experiments about interstellar ice analogues in conditions as close as possible to the interstellar environment. It is this joint research that is presented in the following pages, from the initial theoretical work to the final experimental confirmation, focused on the adsorption of two couples of isomers, namely: acetic acid 
(AA)/methyl formate (MF) and ethanol (EtOH)/di-methyl ether (DME) that appeared as exceptions to the minimum energy principle(MEP) (Lattelais et al. 2009).

The theoretical approach is presented in Sect. 2, starting with a brief outline of the methods used in which we discuss the main features of the calculations. The results are then reported for the adsorption of AA/MF and EtOH/DME on crystalline ice. The temperature programmed desorption (TPD) experiments are presented in Sect. 3, focusing on the material relevant to the present study. A complete description of the experimental setups is well beyond the current objective and will be given in two forthcoming papers, together with the full set of data obtained by each experimental team (LERMA for EtOH/DME and LPMAA for AA/MF). The adsorption energies of the same species were then estimated from the TPD curves obtained for crystalline deposits. The discussion of the consequences of our research is presented in Sect. 4.

\section{Theoretical approach}

Since the astrophysical conclusions that can be drawn from the present calculations rely essentially on relative adsorption energies, particular attention was given to their calculation (a more detailed account of the computational schemes is presented elsewhere). Briefly, the strategy is based on the density functional theory (DFT), which is totally justified in terms of the strength of the interactions considered, namely, hydrogen bonding between the adsorbates and the ice.

\subsection{Computational background}

The modelling of bulk and surface ice structure and reactivity has attracted some attention in the past few years (Hirsch \& Ojamae 2004; Kuo \& Singer 2003; Casassa \& Pisani 2002). It provides, together with our own experience (Casassa et al. 2005; Calatayud et al. 2003), the basis for the present study. Levering on the preceding results, we took a periodic representation of solid water in the form of hexagonal ice composed of bi-layers of water molecules. Among the different structures possible, we considered only the apolar variety. On the one hand, it seems the most plausible arrangement according to the crystallisation conditions in the ISM and on the other hand, it is computationally justified because only apolar structures can generate slabs that are stable (Bussolin et al. 1998), reproduce the bulk properties, and at their surfaces have a balanced distribution of alternate hydrogen and oxygen sites (for a complete discussion see Casassa et al. 2005).

The model unit cell $1 \mathrm{G}$ contains two bilayers (Fig. 1), i.e., an elementary volume whose replication in three spatial directions creates a material of infinite dimensions. This volume is defined by three structural parameters $a, b$, and $c$. Here, the basal plane dimensions (corresponding to the surface interacting with the adsorbate) are $a=7.145 \AA$ and $b=8.73 \AA$. The third dimension of the cell was determined so that there is no interaction between molecules generated by a translation perpendicular to the ice surface. A value of $c=30 \AA$ was used thereafter for all calculations.

Another consideration required is the low abundance of the interstellar species. It follows that lateral interactions between adsorbates generated by horizontal translations of the unit cell have to be eliminated. We verified that an inter molecular distance larger than $14 \AA$ was necessary, which led us to double the dimension of the cell in the two directions $a$ and $b$ of the plane while retaining the height of $30 \AA(2 \mathrm{G})$.

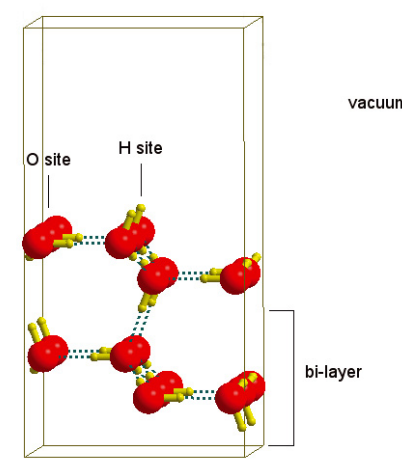

(a) (b)

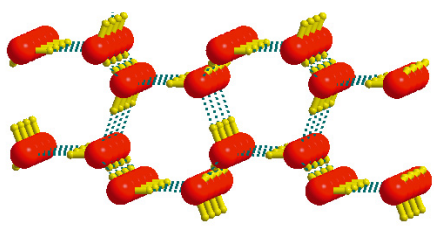

Fig. 1. Views of hexagonal apolar ice: unit cells $1 \mathrm{G}$ a) and $2 \mathrm{G}$ b) with two bi-layers. Color code: $\mathrm{O}$ atoms in red; $\mathrm{H}$ atoms in yellow; hydrogen bonds in dotted lines.

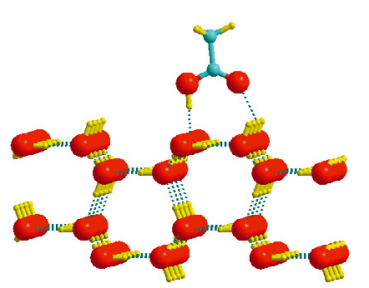

(a)

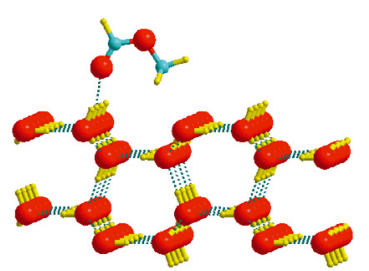

(b)
Fig. 2. Adsorption structures of acetic acid a) and methyl formate b) on the ice surface (unit cell $2 \mathrm{G}$ ). Color code: $\mathrm{O}$ atoms in red; $\mathrm{H}$ atoms in yellow; $\mathrm{C}$ atoms in blue; hydrogen bonds in dotted lines.

Practically, the calculations were done with the VASP package (Kresse \& Hafner 1994). This code was designed to study periodic systems, using pseudo-potentials and a plane wave basis set. As in previous work, the PW91 functional (Perdew et al. 1992) was employed. Core electrons were described by ultrasoft pseudo-potentials (Kresse \& Hafner 1994) and valence electrons by the plane wave basis set with a kinetic energy cut-off at $500 \mathrm{eV}$. The use of plane waves has the additional advantage of removing the well-known problems of the basis set superposition errors (BSSE), which are common in the treatment of weakly interacting systems when using finite basis sets (Boys \& Bernardi 1970) and whose correcting schemes are always a matter of discussion (Lendvay \& Mayer 1998).

\subsection{Acetic acid versus methyl formate}

It can be seen in Fig. 2 that AA and MF have two different types of interactions with the ice surface.

i) Acetic acid is adsorbed by means of two hydrogen bonds that link the - $\mathrm{COOH}$ group to the ice, one between the hydroxylic hydrogen of $-\mathrm{OH}$ and an oxygen site of the ice, the other between the $>\mathrm{C}=\mathrm{O}$ oxygen and a hydrogen site of the ice. Furthermore, the two oxygen atoms of - $\mathrm{COOH}$ are well positioned on top of the sites. This leads to a very strong interaction ${ }^{1} \sim 17.2 \mathrm{kcal} \mathrm{mol}^{-1}$ that fixes acetic acid as a conjugated bridge between two adjacent sites of the surface.

ii) Methyl formate is adsorbed by means of only one hydrogen bond. This involves a weaker interaction $\sim 7.3 \mathrm{kcal} \mathrm{mol}^{-1}$

${ }^{1}$ For convenience: $1 \mathrm{kcal} \mathrm{mol}^{-1}=350 \mathrm{~cm}^{-1}=500 \mathrm{~K}$. 


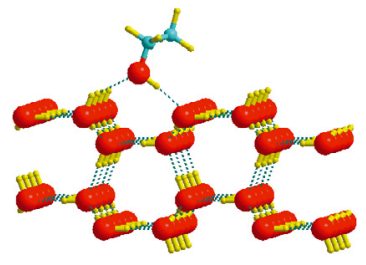

(a)

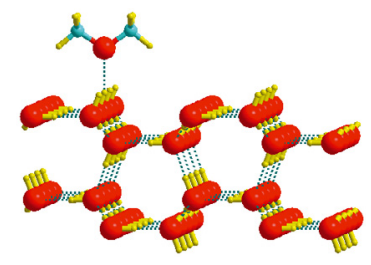

(b)
Fig. 3. Adsorption structures of ethanol a) and dimethyl-ether b) on the ice surface (unit cell $2 \mathrm{G}$ ). Color code: $\mathrm{O}$ atoms in red; $\mathrm{H}$ atoms in yellow; $\mathrm{C}$ atoms in blue; Hydrogen bonds in dotted lines.

between one oxygen atom of the adsorbate and a single hydrogen site on the ice surface.

A comparison of the adsorption energies shows that acetic acid is more strongly attached to the crystalline ice than methyl formate. The energy difference of $\sim 10 \mathrm{kcal} \mathrm{mol}^{-1}$ is consistent with the number and strengths of the hydrogen bonds predicted for each species.

\subsection{Ethanol versus dimethyl ether}

As seen previously for $\mathrm{AA}$ and $\mathrm{MF}$, the couple of isomers $\mathrm{EtOH} / \mathrm{DME}$ have two different types of interactions with the ice surface (Fig. 3).

i) Ethanol is adsorbed by means of two hydrogen bonds, one between the hydroxylic hydrogen of EtOH and an oxygen site of the ice, the other between an oxygen electron pair of $\mathrm{EtOH}$ and a hydrogen site of the ice. It is a strong interaction $\sim 13.5 \mathrm{kcal} \mathrm{mol}^{-1}$ that fixes ethanol as a bridge between two adjacent sites of the surface.

ii) Dimethyl ether is adsorbed by means of one hydrogen bond only. It is a weaker interaction $\sim 8.5 \mathrm{kcal} \mathrm{mol}^{-1}$ between the oxygen atom of DME and a single hydrogen site on the ice surface.

A comparison of the adsorption energies shows that ethanol is more strongly attached to the crystalline ice than the dimethyl ether. The energy difference of $\sim 5 \mathrm{kcal} \mathrm{mol}^{-1}$ is consistent with the number of hydrogen bonds implied for each species.

\section{Experimental approach}

The adsorption of small organic molecules on water ice has been experimentally studied in ultrahigh vacuum (UHV) setups (base pressure of $\sim 10^{-10}$ Torr). Experiments dedicated to the acetic acid (AA) and methyl formate (MF) adsorptions have been completed with the SPICES setup (Surface Processes and ICES) at the LPMAA-UPMC, whereas ethanol (EtOH) and dimethyl ether (DME) have been studied with the FORMOLISM setup (FORmation of MOLecules in the InterStellarMedium) at the LERMA/LAMAp-Université Cergy-Pontoise. Both experiments are based on the temperature programmed desorption (TPD) technique, which we now describe.

The molecular ices are grown in situ on a polycrystalline gold substrate kept at low temperature by means of a close cycle He-compression cryostat. Molecules are deposited on the cold surface for a given time, which results in an exposition usually expressed in Langmuir (L) where $1 \mathrm{~L}$ corresponds to a partial pressure of $10^{-6}$ Torr during $1 \mathrm{~s}$. Expositions can then be calibrated in coverage, expressed in the case of water in terms of bilayers (BL) or in the case of other molecules in terms of monolayers (ML). They are both associated with a saturating first layer of molecules on the substrate. In the present experiments, water ices are first grown directly onto the gold substrate. The ice structure is controlled by adjusting the sample temperature during deposition. The growth at $80 \mathrm{~K}$ is expected to result in an amorphous water ice, while crystalline ice is obtained by dosing the water on the surface kept at $140 \mathrm{~K}$, or by heating an amorphous water ice at $150 \mathrm{~K}$ for a short time (Rowland et al. 1991; Dohnalek et al. 1999; Kimmel et al. 2001). Submonolayers ( $<1 \mathrm{ML})$ of the organics are then obtained by dosing the molecules on top of the resulting ice, which is maintained at $80 \mathrm{~K}$. Water has been purified by several freezepump-thaw cycles prior to use. The organic products studied are industrial products of high purity: AA 99.99\% (Sigma-Aldricht), MF > 99.8\% (Fluka), EtOH 100\% (Prolabo), and DME 99.9\% (Fluka).

TPD experiments are performed using a quadrupolar mass spectrometer (QMS) to monitor the desorbing species, while resistively heating the sample with a controlled linear ramp. TPD spectra are acquired with slow heating rates ranging from 1 to $10 \mathrm{~K} \mathrm{~min}^{-1}$. Several masses are continuously probed, allowing a simultaneous record of both the organic overlayers and the water ice desorption.

TPD of MF and AA were carried out in the SPICES setup by acquiring the signal of the ions $\mathrm{H}_{3} \mathrm{CO}^{+}(m=31$ a.m.u.) and $\mathrm{H}_{3} \mathrm{C}_{2} \mathrm{O}^{+}$( $m=43$ a.m.u.) respectively, which are the most intense ionic fragment signals from the electron impact ionization of the neutral molecules ${ }^{2}$.

TPD of EtOH and DME are carried out with the FORMOLISM setup (at a ionization energy of $30 \mathrm{eV}$ ) by acquiring the signal of the ions $\mathrm{H}_{2} \mathrm{COH}^{+}$( $m=31$ a.m.u.) and $\mathrm{H}_{3} \mathrm{COCH}_{2}^{+}$( $m=45$ a.m.u.), respectively.

In addition, TPD spectra associated with other dominant fragments are also recorded for confirmation and showed no differences from those obtained with the most intense fragments: $m=43,45$, and 60 for AA, $m=31$ and 60 for MF, $m=27,29$, 45 , and 46 for EtOH and $m=15,29$, and 46 for DME. Water desorption is probed by monitoring the signal of the $\mathrm{H}_{2} \mathrm{O}^{+}$and $\mathrm{HO}^{+}$ions.

\subsection{Acetic acid versus methyl formate}

Figure 4 shows the TPD curves of $\mathrm{HCOOCH}_{3}(0.3 \mathrm{~L})$ and $\mathrm{CH}_{3} \mathrm{COOH}(0.6 \mathrm{~L})$ deposited onto $15 \mathrm{BL}$ of water ice at $80 \mathrm{~K}$. Previous experiments, which had been performed at different organic exposures, allowed us to distinguish the first monolayer feature from the multilayer ones. In the present study, the exposures of AA and MF used correspond to a submonolayer coverage onto the ice surface (Bertin et al. 2011). In Fig. 4a, both the desorption curves of $\mathrm{HCOOCH}_{3}$ and $\mathrm{CH}_{3} \mathrm{COOH}$ from crystalline ice surface exhibit a single peak feature. However, the behaviors of these species markedly differ. $\mathrm{HCOOCH}_{3}$ fully desorbs from the ice surface between $115 \mathrm{~K}$ and $135 \mathrm{~K}$, which is lower in temperature than the sublimation of the pure crystalline water substrate, shown in Fig. 4b for comparison. In contrast, $\mathrm{CH}_{3} \mathrm{COOH}$ is released in the gas phase from 150 to $160 \mathrm{~K}$, i.e. in the same temperature range as the crystalline ice desorption. Since the two isomers are deposited as submonolayers, the differences observed between the two species are related to differences in the interaction energies between the molecules and the ice surface. These curves therefore indicate that MF is less

${ }^{2}$ See: http://webbook.nist.gov/chemistry 


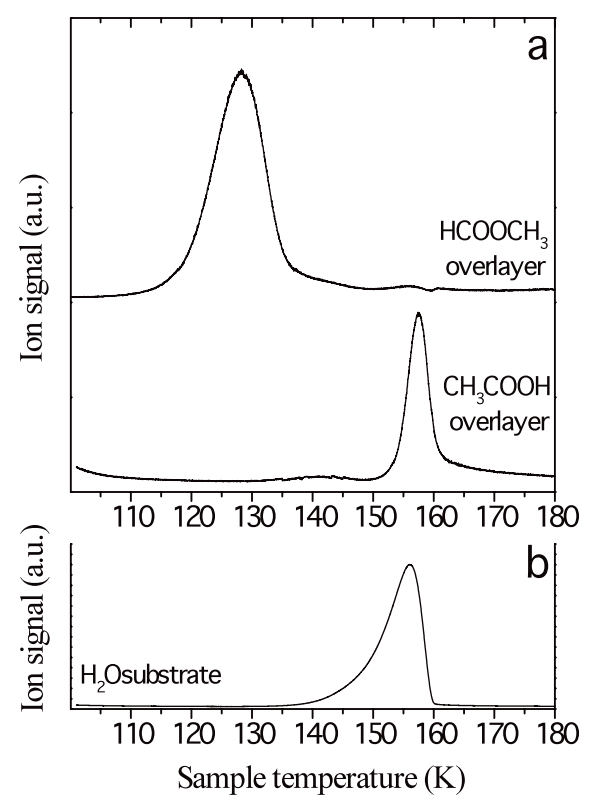

Fig. 4. Thermal desorption curves of submonolayer coverages of methyl formate and acetic acid deposited on top of a $15 \mathrm{BL}$ crystalline $\mathrm{H}_{2} \mathrm{O}$ substrate at $80 \mathrm{~K}$. The temperature ramp is $1 \mathrm{~K} \mathrm{~min}^{-1}$. The desorbing species signal have been detected by monitoring the signal of the most intensive ionic fragments, i.e. a) $\mathrm{H}_{3} \mathrm{CO}^{+}$for methyl formate, $\mathrm{H}_{3} \mathrm{C}_{2} \mathrm{O}^{+}$ for acetic acid and b) $\mathrm{H}_{2} \mathrm{O}^{+}$for water.

tightly bound to the water surface than AA. In the first case, the methyl formate monolayer desorption occurs well before the sublimation of the crystalline ice substrate, allowing the estimation of its adsorption energy by analyzing the TPD curve following a method described later. In the latter case, the simultaneous desorption of the acetic acid and the water strongly suggests that the release in the gas phase of the AA is induced by the sublimation of the substrate underneath. This prevents the extraction of the adsorption energy of AA on water from the analysis of the TPD curves. However, this adsorption energy has to be at least equal to the cohesion energy of the crystalline water ice, which has been evaluated on $\mathrm{Au}$ to be $\sim 11.5 \mathrm{kcal} \mathrm{mol}^{-1}$ (Fraser et al. 2001).

To estimate the adsorption energy of the $\mathrm{HCOOCH}_{3}$ molecules on the ice surface, we assume the desorption of the submonolayer of MF to be a first-order kinetic process following the Polanyi-Wigner equation

$\phi_{\mathrm{d}}=-\frac{\mathrm{d} n}{\mathrm{~d} t}=v(n) n \mathrm{e}^{-\frac{E_{\mathrm{ads}}(n)}{k T}}$,

where $E_{\text {ads }}$ is the adsorption energy, $T$ the sample temperature, $\phi_{\mathbf{d}}$ the desorbing flux at $T, t$ the time, $v$ a pre-exponential factor, $k$ the Boltzmann constant, and $n$ the amount of adsorbed molecules at $T$. By approximating the pre-exponential factor to be independent of both the coverage and the energy, one can invert this equation, giving the adsorption energy as a function of the amount of remaining molecules (DeJong \& Niemantsverdriet 1990; Dohnalek et al. 2001)

$E_{\mathrm{ads}}=-k T \ln \left(\frac{\phi_{\mathrm{d}}}{n v}\right)$.

One can then extrapolate the linear behavior of the adsorption energy with the amount of remaining molecules to obtain a rough estimation of $E_{\text {ads }}$ in the $n=0$ limit (single molecule adsorption on the ice surface). For a typical range of

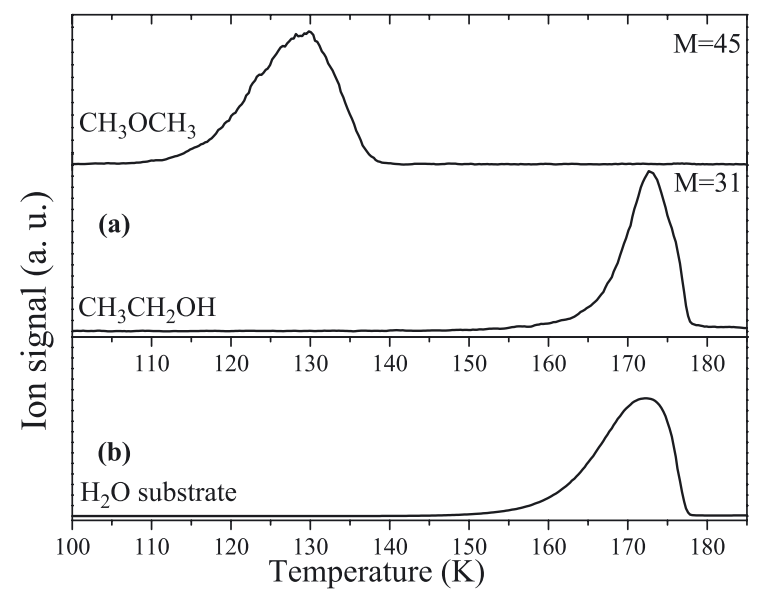

Fig. 5. Thermal desorption curves of submonolayer coverages of dimethyl ether and ethanol deposited on top of a $160 \mathrm{BL} \mathrm{H}_{2} \mathrm{O}$ substrate at $70 \mathrm{~K}$. The temperature ramp is $10 \mathrm{~K} \mathrm{~min}^{-1}$. The desorbing species signal were recorded by monitoring the signal of the most intensive ionic fragments, i.e. a) $\mathrm{H}_{5} \mathrm{C}_{2} \mathrm{O}^{+}$for dimethyl ether, $\mathrm{H}_{3} \mathrm{CO}^{+}$for ethanol and b) $\mathrm{H}_{2} \mathrm{O}^{+}$for crystalline water.

values for $v$ in a first-order process $\left(10^{13}-10^{15} \mathrm{~s}^{-1}\right.$ ) (DeJong \& Niemantsverdriet 1990), we estimate an adsorption energy of $8.9 \pm 1.0 \mathrm{kcal} \mathrm{mol}^{-1}$ for MF on the crystalline water surface.

\subsection{Ethanol versus dimethyl ether}

The spectrum in Fig. 5a shows the TPD curves of $\mathrm{CH}_{3} \mathrm{OCH}_{3}$ $(0.42 \mathrm{ML})$ and $\mathrm{CH}_{3} \mathrm{CH}_{2} \mathrm{OH}(0.37 \mathrm{ML})$ deposited at $70 \mathrm{~K}$ on $160 \mathrm{ML}$ of water ice $\left(1\right.$ monolayer $=10^{15}$ molecules $\left.\mathrm{cm}^{-2}\right)$. Figure $5 b$ shows the TPD curves of water. We note that the water desorption curve reaches a maximum at higher temperature in Fig. 5b than in Fig. 4b (170 K and $155 \mathrm{~K}$, respectively). This is expected for zero order-desorption because a larger ice thickness is used in Fig. 5b than in Fig. 4b (160 ML and 15 ML, respectively). Furthermore, this effect is also enhanced by the higher heating rate adopted for the TPD in Fig. $5 b(10 \mathrm{~K} / \mathrm{min}$ for EtOH versus DME and $1 \mathrm{~K} / \mathrm{min}$ for $\mathrm{AA}$ versus $\mathrm{MF}$ ).

Several experiments were carried out with different exposures to differentiate the features of the first monolayer from those of multilayers. In this paper, the exposures of DME and EtOH correspond to a submonolayer coverage of the ice substrate. In Fig. 5a, the desorption curves of DME and EtOH from the crystalline ice surface display only one desorption peak. However, it is clear that these molecules have different desorption characteristics. DME desorbs from the ice between $112 \mathrm{~K}$ and $138 \mathrm{~K}$, which are lower temperatures than observed for the sublimation of the crystalline ice substrate (see Fig. 5b for comparison). In contrast, EtOH desorbs between $160 \mathrm{~K}$ and $178 \mathrm{~K}$, i.e. in the same range as crystalline ice. This difference is caused by the different interaction energies between these molecules and the ice surface, and it can be concluded that DME is less strongly attached to the surface than EtOH. Using the kinetic model as described in the previous section, we can estimate an adsorption energy of $8.1 \pm 1.0 \mathrm{kcal} \mathrm{mol}^{-1}$ for DME on the crystalline water surface. As seen previously in the case of acetic acid, the simultaneous desorption of $\mathrm{EtOH}$ and water ice prevents us from being able to calculate the adsorption energy of EtOH from the analysis of the TPD curves. It can only be said that, as for acetic acid, it has to be at least equal to 
the $\sim 11.5 \mathrm{kcal} \mathrm{mol}^{-1}$ cohesion energy of crystalline water ice (Fraser et al. 2001).

\section{Discussion and concluding remarks}

The goal of the present work was to investigate whether adsorption at the surface of interstellar ices could affect the abundances observed in the surrounding gas phase. To this end, we have assumed isomers of complex organic molecules can provide invaluable information. In a previous study, we found indeed that, when several isomers of same generic formula are identified, the most stable one was the most abundant. In addition, the abundance ratio of the most stable isomer to the other isomers seemed to be directly related to their energy difference. What can be regarded as a minimum energy principle (MEP) (Lattelais et al. 2009), is verified in molecular clouds, hot cores/corinos, photodissociation regions, and asymptotic giant branch stars. We have proposed that the few exceptions encountered could be rationalized either by the existence of different routes of formation with no intermediate processes in common and/or have a special type of depletion on the grains of one isomer with respect to the others. It is the last option that we have addressed here for the examples of acetic acid and ethanol.

Acetic acid has been observed to be between $1 / 10$ to $1 / 100$ less abundant than methyl formate (Mehringer et al. 1997; Remijan et al. 2002, 2003; Cazaux et al. 2003), whereas the abundance of ethanol has been found to be between 0.3 and 3 times that of dimethyl ether (Millar et al. 1988; Ikeda et al. 2001; Nummelin et al. 1998a; White et al. 2003). The contradiction with the MEP comes from the relative stabilities of these isomers. High level ab-initio coupled cluster calculations with large correlation consistent basis sets ${ }^{3}$ have shown (Lattelais et al. 2009) that acetic acid is more stable than methyl formate by $\sim 17 \mathrm{kcal} \mathrm{mol}^{-1}$ and ethanol more stable than di-methyl ether by $\sim 12 \mathrm{kcal} \mathrm{mol}^{-1}$.

The present study shows that both theory and TPD experiments agree in terms of the relative adsorption energies of the different species whenever measurement is possible:

$E_{\text {ads }}\left(\mathrm{HCOOCH}_{3}\right)=9.2 \mathrm{kcal} \mathrm{mol}^{-1}$ (theory);

$8.9 \pm 1.0 \mathrm{kcal} \mathrm{mol}^{-1}$ (TPD);

$E_{\text {ads }}\left(\mathrm{CH}_{3} \mathrm{OCH}_{3}\right)=8.4 \mathrm{kcal} \mathrm{mol}^{-1}$ (theory);

$8.1 \pm 1.0 \mathrm{kcal} \mathrm{mol}^{-1}$ (TPD).

For the isomers that desorb with the ice one has

$$
E_{\text {ads }}\left(\mathrm{CH}_{3} \mathrm{COOH}\right)=16.2 \mathrm{kcal} \mathrm{mol}^{-1} \text { (theory), }
$$$$
E_{\text {ads }}\left(\mathrm{CH}_{3} \mathrm{CH}_{2} \mathrm{OH}\right)=13.5 \mathrm{kcal} \mathrm{mol}^{-1} \text { (theory), }
$$

which are higher than the cohesion energy of crystalline water ice $\left(\sim 11.5 \mathrm{kcal} \mathrm{mol}^{-1}\right)$. These adsorption energies are related to the number of hydrogen bonds between the substrates and the ice surface. The strength of the interactions depending on the nature of each individual species and not on their intrinsic stabilities, the relative abundances observed in the gas phase clearly differ from the relative abundances of the same molecules on the ices surfaces. This could, at least partially, explain the differences to the MEP.

In this context, a spatial mapping of different hot cores sources should give valuable information about the relevance of differential adsorption to the gas phase abundances. The more weakly bonded molecules should indeed be observed in

\footnotetext{
${ }^{3} \operatorname{CCSD}(\mathrm{T}) / \mathrm{cc}-\mathrm{pVQZ}$.
}

larger quantities in the outer (colder) regions, whereas strongly bonded species should be observed preferentially in the inner (warmer) parts. Observations in the Sagittarius B2 (N-LMH) from Hollis et al. suggest however that $\mathrm{AA} / \mathrm{MF}$ are both present in the warmer region, the glycoaldehyde being more spatially dispersed (Hollis et al. 2001). This finding tends to indicate that differential adsorption might be almost insignificant in this case. In addition, the efficiency of the chemical formations routes have obviously to be considered when interpreting the relative abundances. For instance, in the case of the AA/MF isomer couple, laboratory experiments have shown that different chemical routes are involved in the formation of each isomer. MF (and to a lower extent glycoaldehyde) can be synthesized from electron or proton irradiation of condensed mixtures of $\mathrm{CO}$ and $\mathrm{CH}_{3} \mathrm{OH}$ (Bennett \& Kaiser 2007; Modica \& Palumbo 2010), whereas the formation of acetic acid involves irradiation of $\mathrm{CH}_{4}: \mathrm{CO}_{2}$ mixed ices, via a different mechanism (Bennett \& Kaiser 2007). Both the chemical mechanism efficiency and the stoechiometry of the ices should have an important impact on the relative amount of isomers formed in the icy mantles. We conclude that other similar studies (based either on spatial mapping or on rotational temperature determination) of various hot cores/corinos and other objects of different types, are needed to determine the environment in which the differential adsorption is or is not a relevant enough phenomenon to explain the MEP exceptions for the gasphase isomer relative-abundances.

In the end, one should stress that differential adsorption between molecules is certainly a more general effect than illustrated here. Interstellar ices obviously play an important role in monitoring the release/trapping of molecules at their surfaces, thus affecting in return the gas phase, as it has been demonstrated for the warm-up phase of hot molecular cores (Garrod \& Herbst 2006).

Acknowledgements. This work was supported by CNRS national program PCMI (Physics and Chemistry of the Interstellar Medium), the Agence Nationale pour la Recherche (contract ANR-07-BLAN-0129), the CM0805The Chemical Cosmos, and special funds of Conseil Régional d'Ile de France (contract SESAME I-07-597R) together with a grant from Conseil Général du Val d'Oise (CGVO95). Part of the calculations were performed using HPC resources from GENCI-CINES (Grant 2009-085128).

\section{References}

Allamandola, L. J., Bernstein, M. P., Sandford, S. A., \& Walker, R. L. 1999, Space Sci. Rev., 90, 219

Bennett, C. J., \& Kaiser, R. I. 2007a, ApJ, 660, 1289

Bennett, C. J., \& Kaiser, R. I. 2007b, ApJ, 661, 899

Bertin, M., Romanzin, C., Michaut, X., Jeseck, P., \& Fillion, J.-H. 2011, J. Phys. Chem. C, submitted

Bossa, J.-B., Borget, F., Duvernay, F., Theulé, P., \& Chiavassa, T. 2008, J. Phys. Chem. A, 112, 5113

Bossa, J.-B., Duvernay, F., Theulé, P., et al. 2009, A\&A, 506, 601

Boys, S. F., \& Bernardi, F. 1970, Mol. Phys., 19, 553

Bussolin, G., Casassa, S., Pisani, C., \& Ugliengo, P. 1998, J. Chem. Phys., 108, 9516

Calatayud, M., Courmier, D., \& Minot, C. 2003, Chem. Phys. Lett., 369, 287

Casassa, S., \& Pisani, C. 2002, J. Chem. Phys., 116, 9864

Casassa, S., Calatayud, M., Doll, K., Minot, C., \& Pisani, C. 2005, Chem. Phys. Lett., 409, 110

Cazaux, S., Tielens, A. G., Ceccarelli, C., et al. 2003, ApJ, 593, L51

Chiavassa, A., Ceccarelli, C., Tielens, A. G., Caux, E., \& Maret, S. 2005, A\&A, 431,547

Dohnalek, J. S., Fischer, W., \& Devlin, K. H. 1999, J. Chem. Phys., 110, 5489

Dohnalek, J. S., Fischer, W., \& Devlin, K. H. 2001, J. Phys. Chem., 105, 3747

DeJong A., \& Niemantsverdriet, M. C. 1990, Surf. Sci., 233, 355

Dulieu, F., Amiaud, L., Congiu, E., et al. 2010, A\&A, 512, A30

Fraser, J. S., Fischer, W., \& Devlin, K. H. 2001, MNRAS, 327, 1165

Garrod, R. T., \& Herbst, E. 2006, A\&A, 457, 927

Gibb, E., Whittet, D., Boogert, A., \& Tielens, A. G. 2004, ApJS, 151, 35 
A\&A 532, A12 (2011)

Greenberg, J. M. 1976, Astrophys. Space Sci., 39, 9

Hirsch, T. K., \& Ojamae, L. 2004, J. Phys. Chem. B, 108, 15856

Hollis, J. M., Vogel, S. N., Snyder, L. E., Jewell, P. R., \& Lovas, F. J. 2001, ApJ, 554, L81

Holtom, P. D., Bennett, C. J., Osamura, Y., Mason, N. J., \& Kaiser, R. I. 2005, ApJ, 626, 940

Kemper, F., Jäger, C., Waters, L. B. M. F., et al. 2002, Nature, 414, 295

Kimmel, J. S., Fischer, W., \& Devlin, K. H. 1999, J. Chem. Phys., 114, 5284

Kresse, G., \& Hafner, J. 1994a, Phys. Rev. B, 49, 14251

Kresse, G., \& Hafner, J. 1994b, J. Phys. Condens. Matter, 6, 8245

Kuo, J. L., \& Singer, S. L. 2003, Phys. Rev. E, 67, 016114

Lattelais, M., Pauzat, F., Ellinger, Y., \& Ceccarelli, C. 2009, ApJ, 696, L133

Lendvay, G., \& Mayer, I. 1998, Chem. Phys. Lett., 297, 365

Mathis, J. S., Rumpl, W., \& Nordsieck, K. H. 1977, ApJ, 217, 425

Mehringer, D. M., Snyder, L. E., Miao, Y., \& Lovas, F. J. 1997, ApJ, 480, L71

Millar, T. J., Brown, P. D., Olofson, H., \& Hjalmarson, A. 1997, A\&A, 480, L71

Modica, P., \& Palumbo, M. E. 2010, A\&A, 519, A22

Molster, F. J., Yamamura, I., Waters, L. B. M. F., et al. 2002, Nature, 401, 563
Perdew, J. P., Chevary, J. Ì., Vosko, S. H., et al. 1992, Phys. Rev. B, 46, 6671 Remijan, A., Snyder, L. E., Liu, S.-Y., Mehringer, D., \& Kuan, Y.-J. 2002, ApJ, 576,264

Remijan, A., Snyder, L. E., Friedel, D. N., Liu, S.-Y., \& Shah, R. Y. 2003, ApJ, 590,314

Rowland, J. S., Fischer, W., \& Devlin, K. H. 1991, J. Chem. Phys., 95, 1378

Sandford, S. A. 1996, Meteorit. Planet. Sci., 31, 449

Schutte, W. A. 1999, Solid Interstellar Matter: the ISO Revolution, ed., L. d'Hendecourt, C. Joblin, \& A. Jones (EDP Sciences), 183

Speedy, J. S., Fischer, W., \& Devlin, K. H. 1996, J. Chem. Phys., 105, 240

Tielens, A. G., \& Allamandola, L. J. 1987, Physical Processes in Interstellar Clouds, ed. G. Morfill, \& M. Scholler, NATO ASI (Kluwer), 333

Watanabe, N., \& Kouchi, A. 2008, Progress in Surface Science, 83, 439

Watanabe, N., Shiraki, T., \& Kouchi, A. 2003, ApJ, 588, L121

Watanabe, N., Nagaoka, A., Shiraki, T., \& Kouchi, A. 2004, ApJ, 616, 638

White, G. J., Araki, M., Greaves, J. S., Ohishi, M., \& Higginbottom, N. S. 2003, A\&A, 407, 589 